\title{
Pulsating Heat Pipes Used for Thermal Control of Electronics on Surveillance Systems
}

\author{
Roger R. Riehl ${ }^{1}$, Liomar de O. Cachuté ${ }^{2}$ \\ National Institute for Space Research, São José dos Campos, SP Brazil, 12227-010
}

\begin{abstract}
Surveillance systems have proven to be important applications for high performance thermal control devices, especially the passive ones using heat pipe technology. With the growing need for heat dissipation presented by this type of system, usually hybrid solutions are designed, which include both liquid cooling and passive two-phase thermal control. This last one is usually applied when the heat source is located far from the heat sink and the use of liquid cooling or any other active thermal control system is not possible due to the lack of available space for integration. Since most of the surveillance systems being designed today require thermal control devices that operate under adverse orientation, some restrictions apply. Therefore, the technology that is currently used and disseminated for aerospace missions can find many other applications on surveillance systems for defense purposes. With severe restrictions regarding available space for integration of common thermal control devices, the design and application of pulsating heat pipes become the most indicated solution for the present investigation. Previous investigations have demonstrated that pulsating heat pipes configured as an open loop can operate on adverse conditions, promoting the heat transport from the source to the sink with good reliability. Based on this fact, this investigation is focused on presenting the thermal management of electronic components of a surveillance system being done by pulsating heat pipes configured as open loops. Despite the relatively high temperature difference between the heat source and sink, the open loop pulsating heat pipe is able to transport the rejected heat from the electronic components to a remote heat dissipation area, while keeping their temperatures within the required range established by the project. The operation of the pulsating heat pipes have proven to be stable and reliable, meeting the project's expectations for thermal control.
\end{abstract}

\section{Nomenclature}

$A=$ Heat transfer area $\left(\mathrm{m}^{2}\right)$

$C_{\text {calc }}=$ Calculated thermal conductance for the $\mathrm{PHP}\left(\mathrm{W} /{ }^{\circ} \mathrm{C}\right)$

$C_{C u}=$ Solid copper bar thermal conductance $\left(\mathrm{W} /{ }^{\circ} \mathrm{C}\right)$

$k_{C u}=$ Thermal conductivity of copper $\left(\mathrm{W} / \mathrm{m}^{\circ} \mathrm{C}\right)$

$L_{\text {eff }} \quad=$ Effective length $(\mathrm{m})$

$Q \quad=$ Heat load (W)

$\eta \quad=$ Ratio of conductance

$T_{\text {cond }} \quad=$ Average temperature at the condenser $\left({ }^{\circ} \mathrm{C}\right)$

$T_{\text {evap }} \quad=$ Average temperature at the evaporator $\left({ }^{\circ} \mathrm{C}\right)$

\section{Introduction}

$\mathrm{P}$ ULSATING heat pipes (PHPs) are two-phase thermal control devices that consist of a simple meandering tube bent with several curves forming several parallel channels, without the presence of a wick structure. The channels are formed from capillary tubing and a working fluid is responsible for acquiring the heat from a source and dissipating it in a sink. This kind of device can be considered a special type of heat pipe and was introduced by Akachi ${ }^{1}$. PHPs can be built as open loops where the tubings' ends are not connected and both ends are pinched off

\footnotetext{
${ }^{1}$ Senior Research Engineer and Faculty, Space Mechanics and Control Division, Av dos Astronautas 1758.

${ }^{2}$ Senior Mechanical Engineer, Space Mechanics and Control Division, Av dos Astronautas 1758.
} 
(Fig. 1a) or as closed loops where the ends are connected (Fig. 1b). Figure 1 attemps to show the slug flow circulation of the working fluid for the two different configurations, but the fluid circulation for the open end configuration is rather difficult to simulate and to represent due to its chaotic behavior Usually, the closed loop configuration includes a check valve to constrain the fluid circulation in one direction ${ }^{2-6}$. PHPs can be applied in several thermal control problems, such as microelectronics cooling, but recently have gained interest in applications such as those for Space, Aeronautics and Surveillance systems used on the ground.

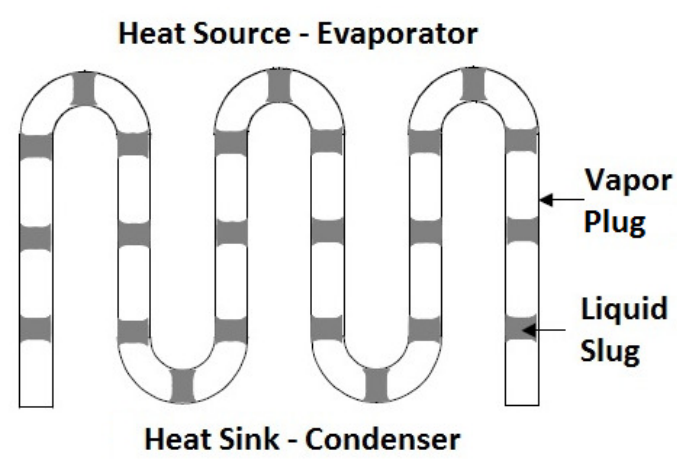

(a) Open loop PHP

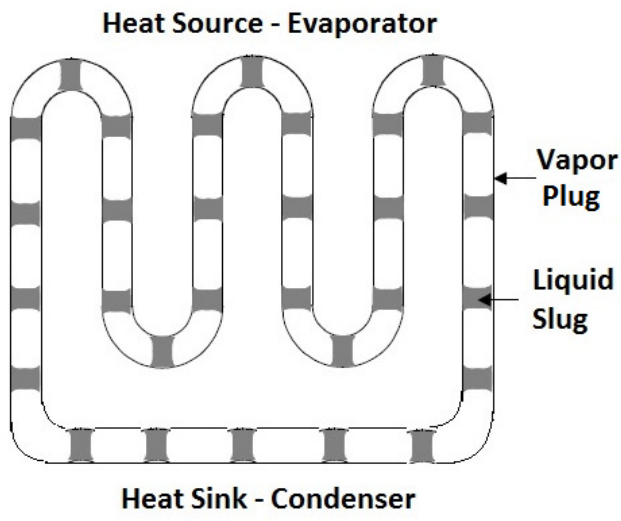

(b) Closed loop PHP

Figure 1. Pulsating heat pipe configurations.

As PHPs operate by means of slug/plug dynamics ${ }^{2}$, several investigations have been performed in order to improve their efficiency, also focusing on working fluids with the presence of solid nanoparticles, which can improve this dynamics as well as the thermal conductivity of the fluid ${ }^{3}$. PHPs have been under investigation in the last years with great development regarding the phenomenon involved in their operation, but further investigations are still required. Delil ${ }^{4}$ has presented a survey on pulsating/oscillating devices suitable to be used in Space and microgravity environments where important contributions to understand such devices were given, indicating the PHPs'capability to operate in Space as well as at high acceleration environments, presenting reliable operation while working at 0 -g, 6-g (anti-gravity) and 12-g (anti-gravity) conditions. For all cases, while operating at low to high acceleration environments, the PHP did not present dryout of its evaporator, showing that this device can operate at levels where regular heat pipes would cease their operation.

Lin et al. ${ }^{5}$ present an experimental investigation on an open loop PHP, where the maximum heat transport capability was investigated, showing the great potential of using PHP as a thermal control device. The first investigations regarding the operation of PHPs were important to guide the future studies and applications, focusing on the use of this technology where the high temperature difference between the heat source and sink was not an issue, as this is a characteristic of this device. The operational limits of closed loop PHPs have been investigated by Yang et $\mathrm{al}^{6}$, indicating that this type of thermal control device can operate at any orientation, even though a loss on its performance can be observed. Recently, Supirattanakul et al. ${ }^{7}$ has presented an investigation where a PHP was used on the performance enhancement of air conditioning systems, showing promising results for such application. Investigations regarding the pressure losses presented in closed loops PHPs were performed ${ }^{8}$, showing that this parameter is the limitation for some applications. Other important investigations regarding the use of PHPs were performed focusing on their improvement upon using the so-called nanofluids ${ }^{9}$, as well as the application of magnetic fields when ferrofluid working fluid is used ${ }^{10}$ and the analysis of the startup on the PHP overall performance ${ }^{11}$. The application of PHPs in Space missions is a recent practice, with promising results already being reported by Iwata et $\mathrm{al}^{12}$, where they have demonstrated the capability of a PHP with a liquid reservoir to perform the thermal control within the temperature range established by the project requirements. Other advances on the thermal control of such a device for Space applications have also been reported with results that indicate robust operation of PHPs in microgravity conditions ${ }^{13}$.

The concern about using PHPs is related to their high temperature difference between the evaporator and condenser sections, which directly affect the thermal conductance for this device. Considering a given thermal design, if this high temperature difference is not an issue, PHPs can be used in any application where a regular heat pipe and/or loop heat pipe is considered, with a much lower cost. As mentioned before, this device can operate in 
zero and high gravity environments and can be used in manned missions when selecting the appropriate working fluid. Proper evaluation and specific designs allow the use of PHPs in extreme environments, just as any other application that requires the use of heat pipes and/or loop heat pipes ${ }^{9,14}$.

Currently, PHPs designs have been conceived and developed by this Institute not only for the purpose as presented in this paper, but also as a thermal control device for electronics in low-orbit satellites, as well as part of anti-icing systems for aircrafts wings ${ }^{14}$.

Focusing on the importance for Defense equipments, this paper is intended to present results related to the application of an open loop PHP designed to meet the requirements for thermal control of electronics components used in surveillance systems. The main objective of this paper is discuss the efforts to evaluate the impacts of PHP orientation, operational temperature range and heat load ability of the PHP to meet heat transport and electronics temperature requirements.

\section{Surveillance System Thermal Control}

A specific design for the surveillance equipment (Defense application) has been conceived to operate in hostile environments where the ambient temperatures can range from -20 to $+50{ }^{\circ} \mathrm{C}$ and humidity levels of up to $95 \%$. In this case, a single-phase thermal control loop has been designed, which presents a forced circulation using a pump to move the working fluid (mixture of water and ethylene glycol) throughout the architecture of the circuit to remove heat from the electronic components, rejecting this heat to the environment by a fan cooling system. A schematic of such arrangement is presented by Fig. 2 .

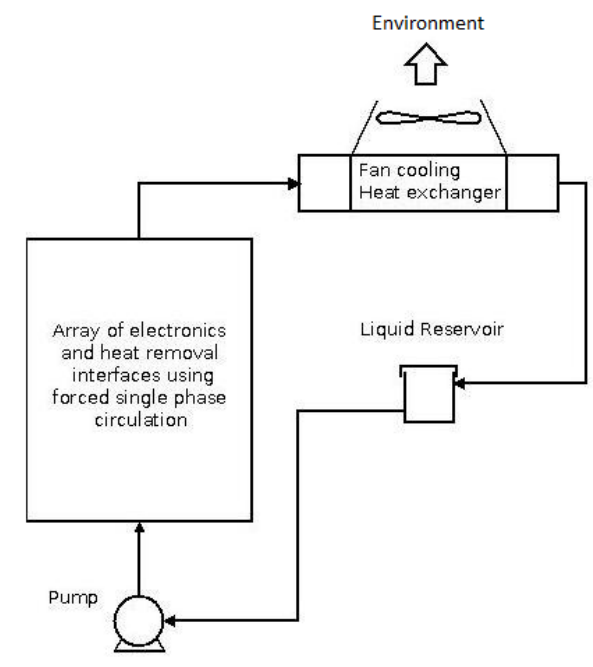

Figure 2. General schematics of the thermal control system arrangement.

Details of the surveillance equipment and its architecture are considered proprietary information and cannot be disclosed at this time. For the sake of the necessary information to perform the adequate analysis, the system's characteristics are:

- Total heat rejection of up to $50 \mathrm{~kW}$ to the environment being composed of the following:

- $47.6 \mathrm{~kW}$ of heat from components with direct contact with the heat sinks;

- $2.4 \mathrm{~kW}$ of heat generated by the so-called class B electronic components.

- Working fluid (50\% water and 50\% ethylene glycol) circulation through $45 \mathrm{~m}$ of tubes, distributed in parallel lines connected by manifolds to the main transport lines, pump, reservoir and fan cooling;

- Maximum required temperature difference between the inlet and outlet of the fan cooling system is $15^{\circ} \mathrm{C}$;

- The working fluid is circulated using a pump and an electronic control sets the pump and fan speeds in order to adjust their operation, according to the working fluid's set point temperature.

Due to the architecture of the surveillance system, some of the electronic equipment (called here as class B) are located far from the main array of electronics and heat removal interfaces, as presented by Fig. 3a. Since the amount of heat dissipated by the class B electronics are highly concentrated and cannot be neglected $(2.4 \mathrm{~kW})$, another 
interface had to be used to connect them to the main heat dissipation interface (the liquid loop). The addition of extra liquid transport lines from the main cooling system would dramatically affect the pumping requirements for this project; therefore a different solution for this problem had to be obtained.

For this specific application, first the use of regular heat pipes was considered to promote the heat transport from the class B electronics to the heat dissipation interface, but certain restrictions applied due to the need of the surveillance system to operate at both gravity assisted and adverse orientations, which would cause the operational failure of the heat pipe. Several heat pipe simulations and tests were performed in order to evaluate their feasibility for this application, but failures that occurred during certain operational conditions let to the decision to avoid their use for this application. Second, loop heat pipes were also considered, the restricted available area for integrating the evaporator and their elevated costs were key factors that eliminated their application to this project.

Based on technical considerations regarding the project's requirements for thermal control, PHP's selected for more detailed evaluation due to their low cost of fabrication, easy integration within the available area to connect the class B electronics to the main heat dissipation interface (sink) and their capability to operate at any orientation ${ }^{9,14}$. Since the main requirement for the class B electronics is to keep their temperatures below $80{ }^{\circ} \mathrm{C}$ at their maximum heat load of $50 \mathrm{~W}$, the high temperature difference observed between the PHP's heat source and sink was not considered a concern. Several activitie, including extensive numerical simulations and experimental tests were performed to develop the PHP architecture that could meet the project's requirements. Promising results were observed that gave confidence to apply such an alternative thermal control device to a very important application.

\section{Pulsating Heat Pipe Design}

The PHPs applied to this project were configured as open loop as they present better operation at any orientation than the PHP's configured as closed loops ${ }^{8,10,14}$. For higher thermal efficiency, copper capillary tubes were used to manufacture the PHPs, with an outer diameter of $3 \mathrm{~mm}$, an inner diameter of $1.6 \mathrm{~mm}$ and a total linear length of $3 \mathrm{~m}$. With this configuration, the PHP provides on its final form $1.2 \mathrm{~m}$ of linear length for the evaporator section, $0.60 \mathrm{~m}$ of linear length for the adiabatic section and $1.2 \mathrm{~m}$ of linear length for the condensation section. In total, 48 open loop PHPs have been designed for this application operating at a maximum temperature of $80^{\circ} \mathrm{C}$, with the class B electronics highest heat dissipation rate of $50 \mathrm{~W}$ each, for a total of $2.4 \mathrm{~kW}$ of heat dissipation capability.

Due to the temperature operation range of the surveillance system, methanol (minimum purity of $99.98 \%$ ) has been selected as the working fluid. The filling ratios of $30 \%$ and $80 \%$ (of the internal volume) were evaluated, in order to select the most appropriate amount of fluid for the highest thermal performance. The filling ratios of 30 and $80 \%$ have been considered in this report as they were already evaluated in previous investigations ${ }^{14}$. It has been shown that the minimum filling ratio for an open loop PHP should be $30 \%$ in order to avoid evaporator dryout at the highest heat loads and the maximum should be $80 \%$ in order to avoid the device's failure at highest heat loads due to excess of liquid. The PHPs were thermally connected to the class B electronics as well as with the main heat dissipation interface with aluminum base heat sink compound, in order to minimize the thermal resistances. Figure 3 a presents the schematics of the PHPs used.

A total of 20 type- $\mathrm{T}$ thermocouples (accuracy of $0.3^{\circ} \mathrm{C}$ at $100^{\circ} \mathrm{C}$ ) were used to measure the temperatures throughout the PHP, as presented by Fig. $3 b$, which were connected to a data acquisition system controlled by LabView. Thermocouples TC-19 and TC-20 were connected to the heat dissipation interface. Readings were taken at each 5 seconds and recorded in a spreadsheet for further analysis. The PHPs' heat sinks were kept at a constant temperature of $20^{\circ} \mathrm{C}$ (evaluation test temperature level requirement) in order to better evaluate the devices' thermal capability and their capacity to meet the project's requirements. For the full qualification and acceptance test procedure, the operational temperature range will be applied, following the guidance given by the standard MILSTD-1540d. To simulate the heat source, a kapton skin heater (280 mm X $25 \mathrm{~mm}, 11.5 \mathrm{Ohms})$ was attached to the evaporation section to deliver the desired heat load, which was controlled by a DC power supply.

\section{Results and Discussion}

Tests were performed to evaluate the PHP capability to handle changes on the heat load applied to the evaporation section as this is an expected operation of the electronic components. It became necessary to investigate at which filling ratio the PHP would present better operation regarding the evaporation section temperature, in order to select the most appropriate amount of liquid to be charged to avoid temperature overshooting with a consequent

shut down of the electronic equipment (set to turn off at $85^{\circ} \mathrm{C}$ ). Also, as mentioned before, the PHP must present a capability to operate at any orientation, as the surveillance system will have its orientation changed depending on its mission. 


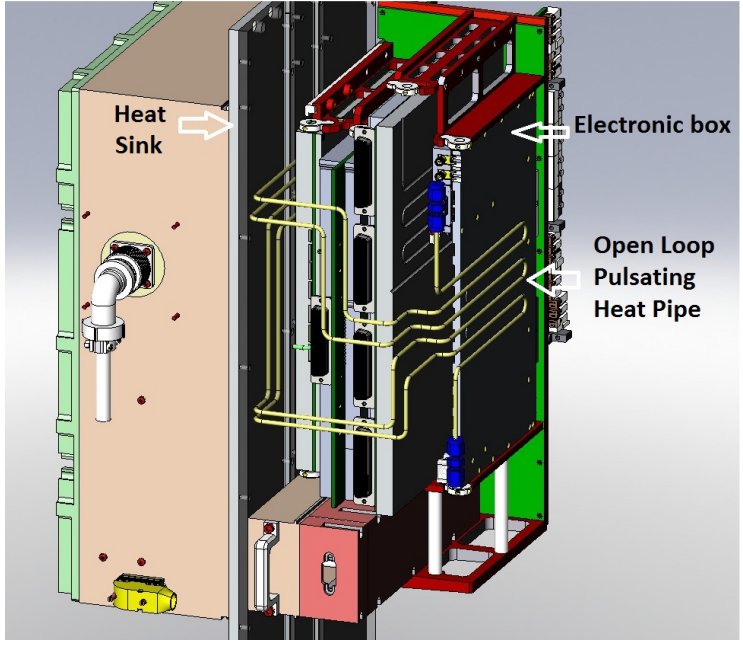

(a) Class B electronic box with the PHP.

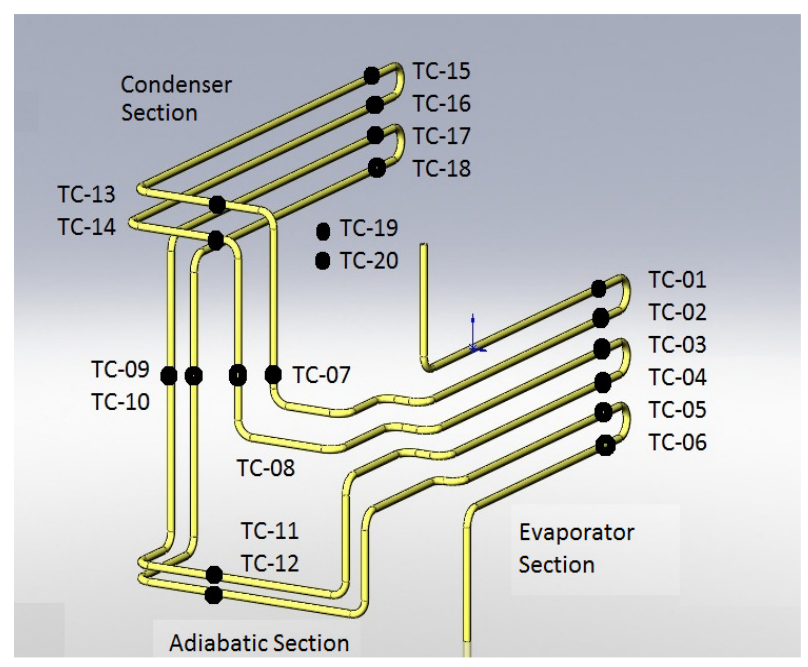

(b) Thermocouples location on the PHP.

Figure 3. Open loop PHP used for the class B electronics cooling and thermocouples location.

Following the requirements for this application, the PHP was tested according to the orientation parameters at which it must operate, and the power to the evaporator was applied to meet some operation conditions of the electronic components. Figure 4 presents the operation of the PHP with a filling ratio of 30\%, operating at both vertical and horizontal orientation, where the temperatures were averaged at all sections. At the vertical orientation, the evaporator section was placed above the condenser section to meet the surveillance system operation at this condition, which characterizes adverse orientation (not gravity assisted operation).

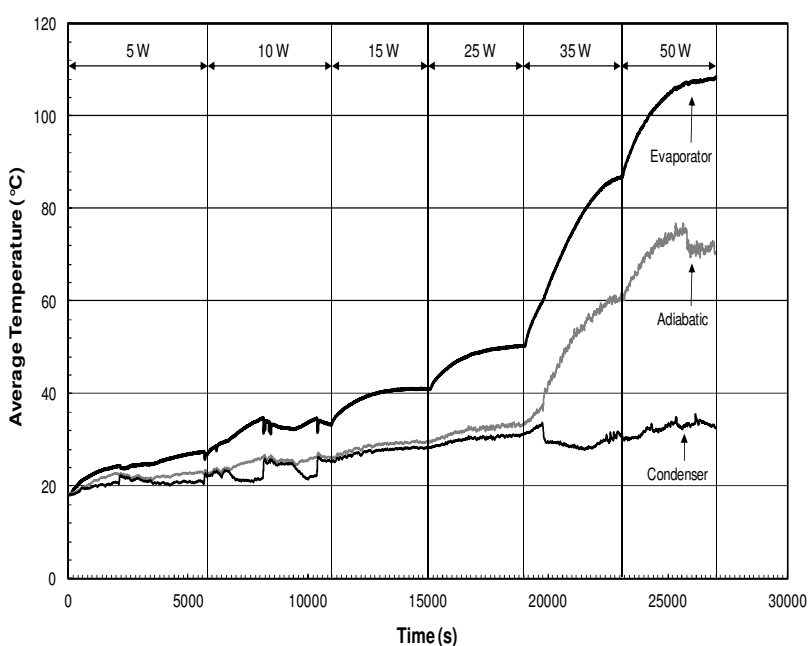

(a) vertical/adverse orientation

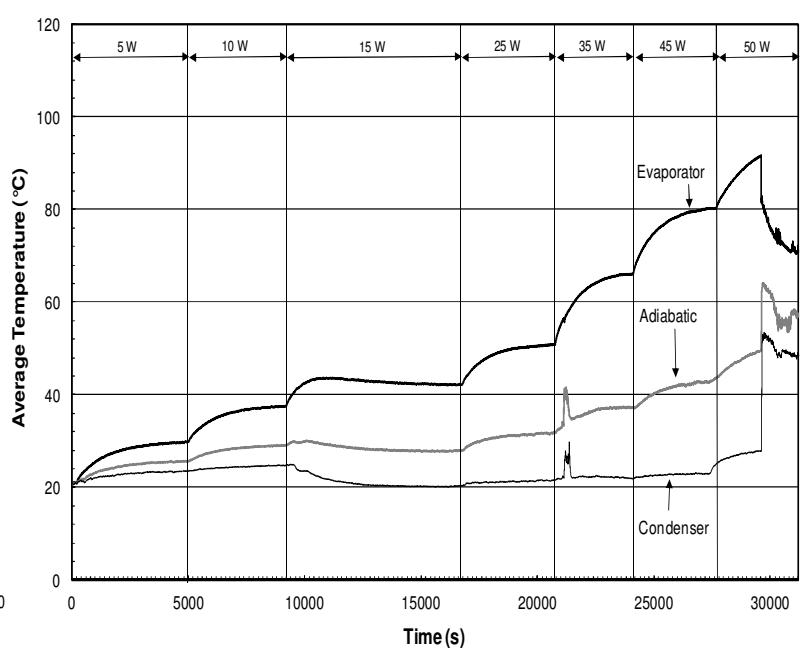

(b) horizontal orientation

Figure 4. PHP operation with filling ratio of $30 \%$.

It is possible to observe that at low power (up to $10 \mathrm{~W}$ ) the PHP operation is believed to be at film evaporation condition. For the vertical/adverse operation, there was an attempt of startup that was not successful at $t=2,160 \mathrm{sec}$ but the startup did occur at $\mathrm{t}=8,125 \mathrm{sec}$ for $10 \mathrm{~W}$. At horizontal orientation, the startup occurred around $\mathrm{t}=10,500 \mathrm{sec}$ for $15 \mathrm{~W}$, where a smooth drop on the temperatures throughout the PHP can be observed. However, the pulsating effects were only visible at higher power (starting at $35 \mathrm{~W}$ and more evident at $50 \mathrm{~W}$ ). The lack of pulsations and a less evident startup were due to the small amount of working fluid applied on this test, even though the operation was observed and it was in accordance to the predictions obtained with simulations. 
From the results presented above, it became clear that additional working fluid was necessary to have the PHP operating at a better condition of temperature observed at the evaporation section at higher power. At vertical/adverse orientation, the PHP did not meet the requirement to operate at $50 \mathrm{~W}$ below $80^{\circ} \mathrm{C}$.

Thus, the filling ratio of $80 \%$ was selected after several numerical/analytical simulations, as well as from previous experimental investigations ${ }^{14}$, because indicated a better operation within the requirements imposed by the project. Figure 5 presents the results related to the vertical/adverse and horizontal orientation tests with this new liquid inventory. For vertical/adverse orientation (Fig. 5a), the PHP presented an operation based on film evaporation up to $35 \mathrm{~W}$ applied to the evaporation section and a clear startup was verified only after increasing the power to $45 \mathrm{~W}$ at $\mathrm{t}=24,400 \mathrm{sec}$. However, special attention must be taken at this operation since the evaporation section temperature was at $90^{\circ} \mathrm{C}$ prior to the startup (effective pulsations), which could compromise the PHP and electronics operation. Such a concern was not considered for the operation at horizontal orientation as the startup (effective pulsations) occurred at $5 \mathrm{~W}$ and a more intense pulsating condition was verified at $35 \mathrm{~W}(\mathrm{t}=21,000 \mathrm{sec})$ and the PHP operation was always below the limit imposed by the evaporation section temperature, as presented by Fig. 5 b.

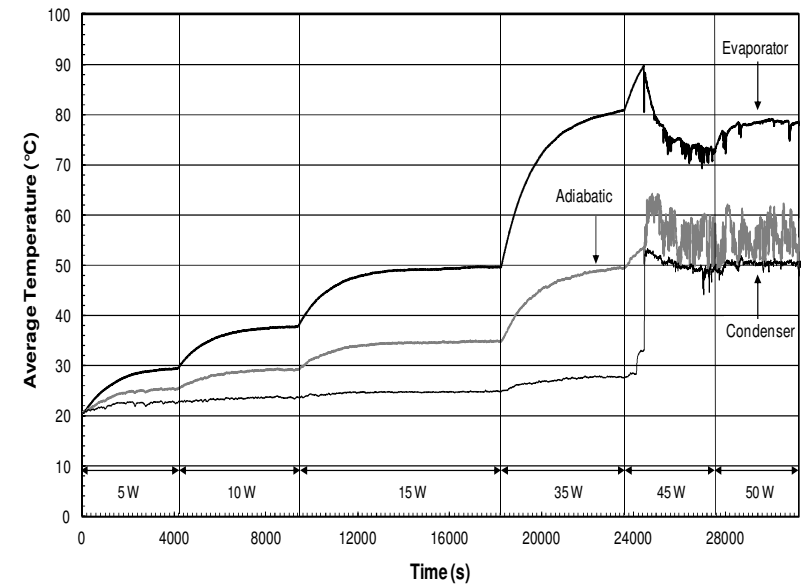

(a) vertical/adverse orientation

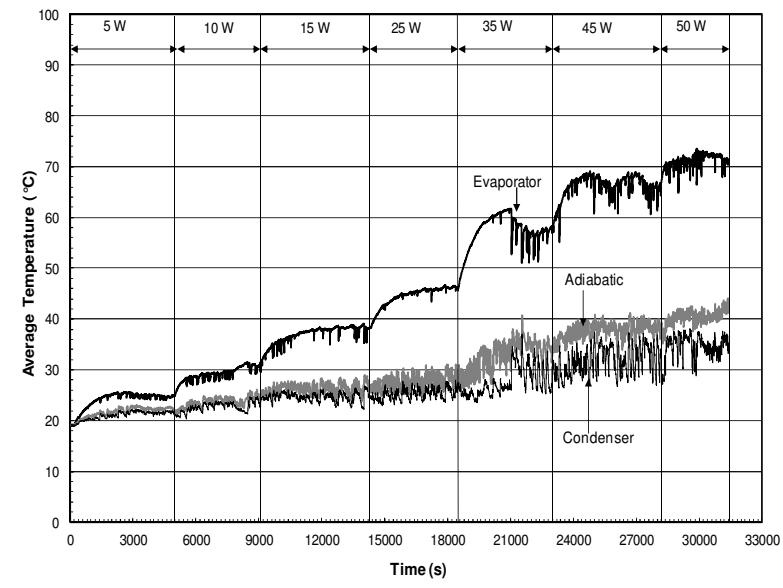

(b) horizontal orientation

Figure 5. PHP operation with filling ratio $80 \%$.

Since more liquid is present at this condition of the PHP, lower temperatures could be observed for all tests and more intense pulsations were also present due to the generation of vapor plugs; this pulsation is a fundamental characteristic of this thermal control device. As mentioned before, despite the high temperature difference observed between the evaporation and condensation sections, this does not represent a concern for this application since the main concern is transferring the heat generated by the class B electronics to the heat dissipation interface, while keeping their temperature below $80^{\circ} \mathrm{C}$.

Such a high temperature difference directly impact on the thermal conductances observed for this type of thermal control device, which is considerably lower than those verified on heat pipes and loop heat pipes. Figure 6 presents the results for the thermal conductance between the evaporation and condensation sections, when the PHP was operating with the filling ratio of $80 \%$, calculated as (with uncertainties below $8 \%$ ):

$$
C_{\text {calc }}=Q /\left(T_{\text {evap }}-T_{\text {cond }}\right),
$$

where it can be observed that while operating at horizontal orientation the results can be more than twice as much as for vertical/adverse orientation.

At both orientations, the PHP was indeed presenting fluid circulation due to either film evaporation at low power (as mentioned above) or pulsating effects caused by the vapor plugs movement inside the tubing (convection). 


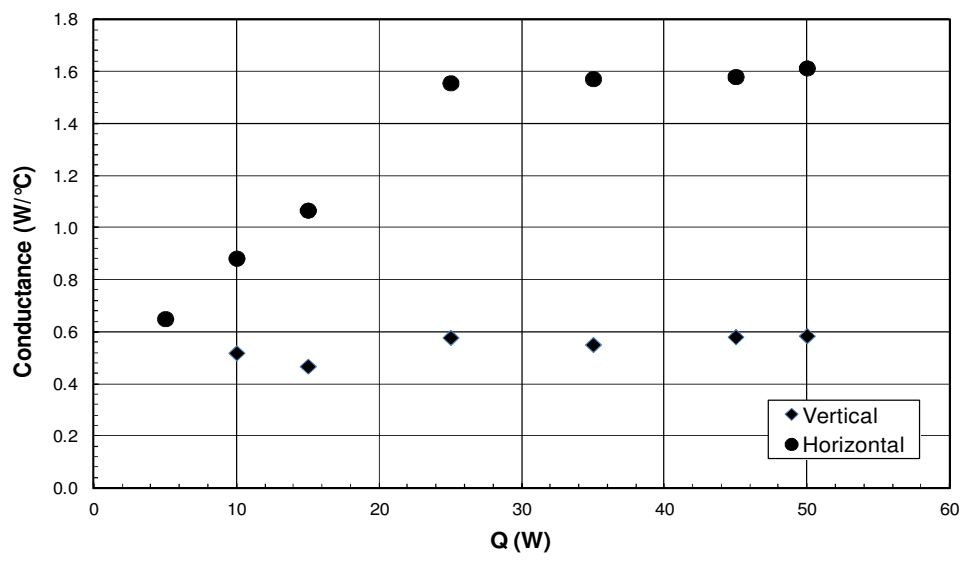

Figure 6. Thermal conductances between the evaporator and condenser.

It becomes important to compare the results regarding the operation of the PHP as if a solid copper bar was used to promote the heat transport, which can have its conductance calculated as

$$
C_{C u}=k_{C u} A / L_{e f f} \cdot
$$

Therefore, the ratio of conductance indicates how many times the PHP filled with methanol is better than a solid copper bar on the heat transfer process, which can be evaluated in terms of

$$
\eta=C_{\text {calc }} / C_{C u} .
$$

From the relation presented by Eq. (3), the PHP operating with filling ratio of $80 \%$, at vertical/adverse orientation would be 4.2 times more efficient than a solid copper bar with the same geometric characteristics ( $3 \mathrm{~mm}$ OD, 5 m linear length, with the same path) used for the PHP. For horizontal orientation, the PHP would be 6.7 times more efficient than a solid copper bar. The presented results show that this thermal control device can be applied for the present project and will be implemented to perform the thermal control of the class B electronics.

Additional developments and tests are ongoing in order to design a PHP that fully meets the project's operating conditions and requirements, and the results obtained so far are promising.

\section{Conclusions}

An investigation on the operation of a PHP designed to operate as a thermal control device in a surveillance system has been presented. This investigation has been carried out in order to verify how this device would operate according to the project's requirements, transferring the heat from a set of electronic equipments that are far from the main heat dissipation interface (heat sink). The main conclusions that can be derived from this investigation are as follows:

a. For both filling ratio tested, the PHP demonstrated its capability to transport the heat with good efficiency for both operation orientations used;

b. For the filling ratio of $30 \%$, the evaporator section temperature at $50 \mathrm{~W}$ was higher than the project's requirements for both orientations tested, which indicates that the PHP does not meet the operation restriction imposed by the project;

c. For the filling ratio of $80 \%$, the PHP demonstrates classical pulsations, provides good heat transport, and meets the temperature requirements for the horizontal orientation; for the vertical/adverse orientation and highest heat loads the performance is sufficient to maintain required temperatures, but reduced flow at moderately high heat loads may allow excessive evaporator temperature to occur. Further development and tests are required at this filling ratio to meet the project's requirements; 
d. Upon continuing the development of the PHP, at both orientations and $80 \%$ of filling ratio, this thermal control device can be used for the purpose that has been designed for, promoting the thermal control of the so-called class B electronics;

e. Even though the PHP presents low thermal conductances, its operation is better than when compared to a solid copper bar. The results show that at vertical/adverse orientation, for filling ratio of $80 \%$, the PHP is 4.2 times better than a solid copper bar and 6.7 times better at horizontal orientation.

Simplicity on manufacturing and integration makes the PHP an important and interesting option for the thermal control of equipments that dissipate low heat and are far from the main heat dissipation interface (heat sink). However, further developments are still required for the PHP project so it can meet the surveillance system's operations requirements for both vertical/adverse and horizontal orientations. Considering the potentials in using this thermal control device for this project, it can be concluded that the PHP will present an additional option for thermal control engineers together with heat pipes and loop heat pipes, as the demand for thermal dissipation increases and new solutions for ground applications are necessary.

\section{References}

${ }^{1}$ Akachi, H., Polasek, F., Stulc, P., "Pulsating Heat Pipes", Proceedings of the $5^{\text {th }}$ International Heat Pipe Symposium, Melbourne, Australia, pp. 208-217, 1996.

${ }^{2}$ Tong, B. Y., Wong, T. N., Ooi, K. T., “Closed-Loop Pulsating Heat Pipe”, Applied Thermal Engineering, 21, 2001, pp. $1845-1862$

${ }^{3} \mathrm{Yu}$, W., Choi, S. U. S., "The role of interfacial layers in the enhanced thermal conductivity of nanofluids: a renovated Maxwell model", Journal of Nanoparticle Research, 5, 2003, pp. 167-171.

${ }^{4}$ Delil, A. A. M., "Pulsating and Oscillating Heat Transfer Devices in Acceleration Environments from Microgravity to Supergravity”, SAE paper \# 2001-01-2240, 2001.

${ }^{5}$ Lin, L., Ponnapan, R., Leland, J., "Experimental Investigation of Oscillating Heat Pipes", AIAA Journal of Thermophysics and Heat Transfer, Vol. 15, No. 4, pp. 395-400, 2001.

${ }^{6}$ Yang, H., Khandekar, S., Groll, M., "Operational Limit of Closed Loop Pulsating Heat Pipes", Applied Thermal Engineering, 28, 2008, pp. 49-59.

${ }^{7}$ Supirattanakul, P., Rittidech, S., Bubprachot, B., “Application of a Closed-Loop Oscillating Heat Pipe with Check Valves (CLOHP) on Performance Enhancement in Air Conditioning System”, Energy and Buildings, 43, 2011, pp. 1531-1535.

${ }^{8}$ Mameli, M., Marengo, M., Zinna, S., "Numerical Model of a Multi-Turn Closed Loop Pulsating Heat Pipe: Effects of the Local Pressure Losses Due to Meanderings", International Journal of Heat and Mass Transfer 55, 2012, pp. 1036-1047.

${ }^{9}$ Riehl, R. R., Santos, N., "Water-Copper Nanofluid Application in an Open Loop Pulsating Heat Pipe", Applied Thermal Engineering 42, 2012, pp. 6-10.

${ }^{10}$ Liu X., Chen, Y., Shi, M., "Dynamic Performance on Start-Up of Closed Loop Pulsating Heat Pipes (CLPHPs)", International Journal of Thermal Sciences 65, 2013, pp. 224-233.

${ }^{11}$ Taslimifar, M., Mohammadi, M., Afshin, H., Saidi, M. H., Shafii, M. B., "Overall Thermal Performance of Ferrofluidic Open Loop Pulsating Heat Pipe: An Experimental Approach”, International Journal of Thermal Sciences, 65, 2013, pp. $234-241$.

${ }^{12}$ Iwata, N., Ogawa, H., Miyazaki, Y., "Experimental Study of Temperature Controllable Oscillating Heat Pipe in Space Thermal Environment", $40^{\text {th }}$ International Conference on Environmental Systems, paper AIAA 2010-6058.

${ }^{13}$ Iwata, N., Ogawa, H., Miyazaki, Y., "Temperature Controlled Oscillating Heat Pipe", Journal of Thermophysics and Heat Transfer Vol 25, No. 3, 2011, pp.386-392.

${ }^{14}$ Riehl, R. R., "Evaluation of the Thermo-Hydro-Dynamics Behavior of an Open Loop Pulsating Heat Pipe", National Institute for Space Research - Internal R\&D Report, 2003, 40pp. 